\section{P-156 MIXED-METHOD STUDY OF EXERCISE IN ADVANCED CANCER: MULTIPLE VIEWS ON ADHERENCE AND LASTING BEHAVIOUR}

1,2,3Daniel Aze. 'University of Southampton, Southampton, UK; ${ }^{2}$ University Hospita Southampton NHS Foundation Trust, Southampton, UK; ${ }^{3}$ Countess Mountbatten House, Southampton, UK

\subsection{6/bmjspcare-2019-HUKNC.178}

Introduction Research suggests exercise can provide numerous benefits for people with advanced cancer. Reported benefits include improved physical performance (Temel, Greer, Goldberg, Vogel, et al, 2009; Bourke, Doll, Crank, Daley, et al., 2011; Oldervoll, Loge, Lydersen, Paltiel et al., 2011; Quist, Rorth, Langer, Jones et al., 2012; Cheville, Kollasch, Vandenberg, Shen et al., 2013; Cormie, Newton, Spry, Joseph et al., 2013; Litterini, Fieler, Cavanaugh \& Lee, 2013; Jensen, Baumann, Stein, Bloch et al., 2014; Rief, Petersen, Omlor, Akbar et al., 2014; Quist, Adamsen, Rorth, Laursen et al., 2015) improved psychological wellbeing (Quist, Rorth, Langer, Jones et al., 2012; Quist, Adamsen, Rorth, Laursen et al., 2015; Burke, Brunet, Sabiston, Jack et al., 2013; Rief, Akbar, Keller, Omlor et al., 2014), reduced fatigue (Bourke, Doll, Crank, Daley et al., 2011; Cheville, Kollasch, Vandenberg, Shen et al., 2013; Litterini, Fieler, Cavanaugh \& Lee, 2013; Jensen, Baumann, Stein, Bloch et al., 2014; Burke, Brunet, Sabiston, Jack et al., 2013; Rief, Akbar, Keller, Omlor et al., 2014), increased completion of planned treatments (Cheville, Alberts, Rummans, Bashford et al., 2015), and fewer hospital admissions (Cheville, Alberts, Rummans, Bashford et al., 2015).

Much of the research into exercise in advanced cancer has involved supervised sessions in specialist settings (Temel, Greer, Goldberg, Vogel et al., 2009; Oldervoll, Loge, Lydersen, Paltiel et al., 2011; Litterini, Fieler, Cavanaugh \& Lee, 2013; Jensen, Baumann, Stein, Bloch et al., 2014; Quist, Adamsen, Rorth, Laursen et al., 2015; Burke, Brunet, Sabiston, Jack et al., 2013; Cheville, Alberts, Rummans, Bashford et al., 2015). Limited healthcare resources mean exercise interventions delivered under these specialist conditions may not be deliverable across a larger population in standard healthcare settings. Attempts to address this have combined supervised hospitalbased sessions with less resource intensive unsupervised homebased sessions. However, several studies have reported a large drop in adherence during the unsupervised exercise (Quist, Rorth, Langer, Jones et al., 2012; Kuehr, Wiskemann, Abel, Ulrich et al., 2014; Winters-Stone, Dobek, Bennett, Maddalozzo et al., 2014; Winters-Stone, Dobek, Bennett, Dieckmann et al., 2015). Reasons for the decline in adherence to the unsupervised home-based sessions have not been thoroughly investigated.

Aim To investigate factors that influence exercise adherence in a series of supervised exercise classes in a hospice day service, and investigate continuing exercise behaviour after class completion.

Methods and analysis People with advanced cancer $(n=11)$, enrolled in a hospice day care exercise class, took part in semi-structured interviews at two time points. Firstly during the supervised class period, secondly six weeks post class completion. At both time points participants also completed a validated questionnaire (Kirby, Donovan-Hall \& Yardley, 2014) which measured factors perceived to have impeded exercise. All staff who delivered the classes $(n=4)$ also participated in one semi-structured interview.
Interview data will be analysed using the framework approach (Richie \& Spencer, 1994; Smith \& Firth, 2011; Spencer, Ritchie, Ormston, O'Connor et al., 2014) to gain knowledge in relation to the identified study aims. The questionnaire will be analysed using descriptive statistics, to show central tendency and dispersion of factors perceived to have prevented exercise.

Results Data collection has been completed. Data analysis is underway.

Conclusion Findings will create a set of recommendations, for intervention designers, to maximise adherence to unsupervised exercise for people with advanced cancer. The recommendations will intend to be applicable in both clinical practice and research settings.

\section{P-157 KEEP MOVING - 5 DAYS A WEEK GYM SERVICE HELPING PALLIATVE CARE PATIENTS}

Jo Wilson, Chris Smith. Dorothy House Hospice Care, Winsley, UK

\subsection{6/bmjspcare-2019-HUKNC.179}

With the national agenda on palliative rehabilitation and with an increase (up 66\%) in referrals to Dorothy House Physiotherapy team in 2015, the team reviewed their gym sessions and in 2017 opened the gym sessions out to include outpatients, day patients and inpatients. This service is now available five days a week and provides group and individual sessions. Due to the increase in sessions and availability of the gym the contacts have increased 327\% from 17/18-18/19.

The review originated from a Palliative Rehabilitation Steering Group that aimed to address wellbeing, falls prevention and rehabilitation in a safe environment. The ultimate aim was to encourage people to work on what they can do now and be able to move to community leisure facilities as/when they are able.

Benefits

- Partnership working with local leisure providers;

- Eased pressure on Dorothy House Hospice Care community Physios;

- Provided a safe environment for people to build confidence and self-esteem when exercising;

- Monitors and addresses other symptoms such as pain, breathlessness and fatigue;

- Can be accessed by all patients referred to Dorothy House Hospice Care;

- Inclusive use of outcome measures e.g. grip strength are used to monitor patients' physical health.

Issues

- There is not always an appropriate place for patients to move on to;

- Variations in exercise provision in the different counties;

- External communication could be improved to GPs;

- Palliative care means that there often needs to be an option for patients to move between service providers if their symptoms improve or deteriorate. This can be more difficult at times.

The future

- Increased partnership working to ensure that the patients will be able to access exercise facilities and support, no matter what stage of their illness they are in. 
- Dorothy House Hospice Care physio continues to provide specialist treatment and advice for those who need it.

- Consideration of seven-day week support (funding dependent).

\section{P-158 COLLABORATION BETWEEN STARLIGHT REHABILITATION SERVICE AND LOCAL EVERYONE ACTIVE GYM}

Jo Marovitch. Peace Hospice Care, Watford, UK

\subsection{6/bmjspcare-2019-HUKNC.180}

Background The Hospice Rehabilitation Service aims to maintain independence, build confidence, improve strength, reduce fatigue, reduce side effects of treatments, depression and anxiety. The benefits of exercise for people living with long-term conditions, both physically, emotionally and psychologically, are widely recognised.

Aims A local gym agreed to provide a Personal Trainer free of charge to run a group under the guidance of hospice staff. The project aims to:

- Enable and empower active and independent patients to access a local community gym for independent exercise;

- Improve patients' health and wellbeing.

Participating patients would then be offered gym membership at a reduced rate on completion of the programme.

Method Patients set their goals, and hospice staff provided referrals, relevant information and training in rehabilitation principles and fatigue to the Personal Trainer. The patients were taken as a group into the gym environment to access the equipment safely at their own level, building their confidence in small steps. Seven patients were referred, and the Personal Trainer developed programmes suited to individual needs.

Results Four completed (57\%) and reported increased strength, mobility and fitness, weight loss and improved confidence. Two of the group have now taken up gym membership and one has achieved his goal to get back onto the golf course.

Conclusion The programme had a positive impact on our patients' health and wellbeing and the hospice has successfully enabled patients to access community resources. The second cohort of patients have been referred and progress is being monitored.

'Exercise not only changes your body, it changes your mind, your attitude and your mood.'

\section{P-159 IMPROVING ACCESS AND OPPORTUNITIES TO EXERCISE FOR PALLIATIVE PATIENTS}

Colette Parfitt. Marie Curie Hospice, Liverpool, UK

\subsection{6/bmjspcare-2019-HUKNC.181}

Background Research suggests that participating in some form of physical activity improves quality of life and leads to better outcomes for palliative patients. However, evidence suggests that there are variable levels of participation and engagement in physical activity for many reasons. One of the common reasons provided is the reluctance to access mainstream gyms and services due to a perceived lack of support and specialist knowledge of their diagnosis and symptoms. Due to our small team, there are often many challenges to providing our patients with these services.

Aims Our aim was to improve the opportunities for hospice patients to participate in supported gym-based exercise groups. Methods We contacted several universities to establish interest in exercise student placements. We developed links with a local university, arranging clinical placements for six exercise students over an eight week period.

Following an initial physiotherapy assessment, and prescription of appropriate exercises, two students worked together delivering gym sessions. Students were encouraged to progress exercises with physiotherapy support and report any issues back to the therapy team.

Following the eight weeks, feedback was obtained from both students and patients.

Results Results have been positive. The students have enjoyed the experience a clinical placement has offered, and two have since enrolled onto a physiotherapy degree course. Uptake onto the classes and gym sessions was good. As well as reporting enjoyment of the sessions, patients reported improvements in quality of life.

Conclusion Our work has demonstrated that offering exercise services within a hospice is important to palliative patients. Developing relationships with other institutions and exploring the use of different academic students (to complement our physiotherapy student placements) is both resourceful and valuable. Work is ongoing. We hope that by maintaining these links we will provide hospice patients with greater opportunity to access beneficial services for the future.

\section{P-160 A PILOT STUDY TO MEASURE OUTCOMES OF USING A REHABILITATIVE PALLIATIVE CARE PHYSIOTHERAPY SERVICE}

Dawn Tidmarsh, Sarah Bradfield. Garden House Hospice Care, Letchworth, UK

\subsection{6/bmispcare-2019-HUKNC.182}

Background Having in the past two and a half years changed our day service delivery from a traditional day hospice to an out-patients supportive rehabilitative care service we wanted to formally measure the outcomes for patients using our physiotherapy and gym programme. Anecdotally patients reported 'feeling better and fitter' but this wasn't fully captured by reviewing their gym programme alone. We hypothesised that in a group of patients with an overall declining picture of health we needed to look at not only their physical condition, but also the potential mental health benefits of exercise. We believed that even if patients were physically declining, we could use the mental health questionnaire results to encourage and motivate patients to continue with their programmes.

Aims Our aim was to pilot a system whereby patients completed an IPOS, identified a goal they wanted to be able to achieve (e.g. 'to be able to walk to town') and completed an anxiety and depression questionnaire.

Methods From late 2018, existing and new patients to the gym have completed an IPOS, identified a specific and measurable goal and have been asked to complete the PHQ-9 (Depression) and GAD-7 (Anxiety) questionnaires. Patients are then reviewed approximately every 12 weeks. Results of the review and questionnaires are collated and used to identify not only further physiotherapy intervention but whether the 